\title{
The infected hip: A treatment algorithm
}

\author{
Bahar Adeli \\ Rothman Institute, Thomas Jefferson University Hospital \\ Javad Parvizi MD \\ Rothman Institute, Thomas Jefferson University
}

Follow this and additional works at: https://jdc.jefferson.edu/orthofp

Part of the Orthopedics Commons

Let us know how access to this document benefits you

\section{Recommended Citation}

Adeli, Bahar and Parvizi, Javad MD, "The infected hip: A treatment algorithm" (2011).

Department of Orthopaedic Surgery Faculty Papers. Paper 31.

https://jdc.jefferson.edu/orthofp/31

This Article is brought to you for free and open access by the Jefferson Digital Commons. The Jefferson Digital Commons is a service of Thomas Jefferson University's Center for Teaching and Learning (CTL). The Commons is a showcase for Jefferson books and journals, peer-reviewed scholarly publications, unique historical collections from the University archives, and teaching tools. The Jefferson Digital Commons allows researchers and interested readers anywhere in the world to learn about and keep up to date with Jefferson scholarship. This article has been accepted for inclusion in Department of Orthopaedic Surgery Faculty Papers by an authorized administrator of the Jefferson Digital Commons. For more information, please contact: JeffersonDigitalCommons@jefferson.edu. 


\section{Bahar Adeli BS, Javad Parvizi MD, FRCS}

\section{Correspondence to:}

Javad Parvizi MD, FRCS

Professor of Orthopedic Surgery

Rothman Insititute at Thomas Jefferson University Hospital

Philadelphia, PA 19107

Email: parvi@aol.com

\section{Introduction}

In 1979, Sir John Charnley accurately predicted that joint sepsis would be the major hurdle facing the orthopedic community of his future. Indeed, decades later, studies are confirming Dr. Charnley's foresight, as they indicate that $0.7 \%-12 \%$ of primary joint replacement cases result in periprosthetic joint infection. However, large or small the percentage, PJI is presently a devastating risk or reality to TJA patients, and an elaborate obstacle facing clinicians and surgeons around the world.

Periprosthetic joint infection is the leading cause of failure of total knee arthroplasty and the third leading cause of failure of total hip arthroplasty ${ }^{1}$. While a timely and accurate diagnosis in these cases significantly aids in eradicating the infection, the treatment option chosen is arguably most telling indicator of success in overcoming $\mathrm{PJI}^{1}$. Therefore, this critical decision is paramount in not 
only successfully eliminating the infection, but also limiting the likelihood of a reinfection as well as continued challenge for both the patient and the surgeon.

In order to overcome PJI and yield a painless, functioning joint, the most appropriate treatment for each patient should be decided through careful consideration of the interaction which exists between a variety of host factors, organism factors, and surgical factors in each specific case. In North America, the two-stage exchange is currently the most preferred treatment method for chronic PJI. However, surgeons may decide on a more fitting solution based the outcomes of clinical assessments and laboratory results. Further treatment options commonly implemented also include the following: antibiotic suppression alone, debridement in addition to the use of antibiotics, and onestage exchange arthroplasty. Salvage operation such as arthrodesis or above the knee amputation is reserved for patients with failed multiple operations and unreconstructible joint.

Dialogue regarding the treatment of PJI is incomplete without mention of biofilm, the formation of which occludes the course of treatment. Initially requiring only a few microorganisms to adhere to the implant, the generation of mature biofilm can take up to 3 weeks ${ }^{2}$ and serves as a communication pathway for the organism ${ }^{3}$. Impenetrable by the host immune system as well as antimicrobial agents ${ }^{4}$, biofilm not only presents an obvious hurdle in the treatment of PJI, but also remains undetected and often complicates diagnosis.

\section{$\underline{\text { Treatment Options }}$}

\section{Antibiotic Suppression Alone}

This is rarely an option in management of PJI. However, in extremely sick and frail patients who may not tolerate surgical treatment antibiotic suppression may be employed to control infection and prevent systemic progression. For this treatment to have an effect, the prosthesis needs to be well fixed and the organism needs to be susceptible to oral antibiotics.

\section{Irrigation and Debridement}

Although one study indicated that $29 \%$ of THA patients ${ }^{5}$ that had debridement with prosthesis retention resulted in failure ${ }^{6-8}$ surgical debridement with component retention plus antibiotic administration is a viable option in certain scenarios. For example, postoperative patients that present with acute symptoms of infection, up to 48 hours after their procedure ${ }^{9}$, may be a good candidate for this treatment method if there is also an identified, susceptible organism. As shown by a multicenter study conducted by the Periprosthetic Infection Consortium (PIC) ${ }^{10}$, the importance of identifying the specific type of pathogen inflicting the infection should not be minimized, since debridement fails in $60-90 \%$ of cases when the infection is caused by Methicillin Resistant Staphylococcus aureus (MRSA) ${ }^{11}$. Due to such undesirable results, this treatment method may not the best option for patients with MRSA infection even at acute stages. Also, debridement is not recommended in cases where there is a loose prosthesis and inadequate soft tissue coverage ${ }^{9}$. It is important to note that debridement is contraindicated if the time from the primary arthroplasty to the diagnosis of $\mathrm{PJI}$ exceeds 30 days, or if the patient has previously undergone this surgical treatment in the same joint ${ }^{5,12}$. 


\section{One-stage Exchange}

One stage exchange arthroplasty, as the name implies, involves removal of the infected prosthesis through debridement of the joint followed by reimplantation with a new prosthesis. This surgical procedure is more often employed in Europe, with some centers reporting very high success rate for eradication of infection ${ }^{13}$. In North America this procedure is usually employed in elderly, frail patients who may not be able to undergo multiple operations. The success rate of this procedure, and others for that matter, is improved if the patient is not immunocompromised, and there is good soft tissue coverage of the infected joint. In addition susceptibility of the organism also impacts the outcome of this surgical intervention directly.

The obvious advantage of this method is that the patient has a component resection and reimplantation during one surgical procedure. Having one multipart operation such as this, results in a single overall recovery period that is generally shorter. Additionally, and in consequence with having one operation, there is also an estimated, overall decrease in expenses associated with this treatment option. A one-stage exchange procedure is contraindicated, however, when the patient is in poor health or the infection is caused by resistant bacteria. Furthermore, this procedure is inadvisable if the joint cannot be reconstructed adequately due to mechanical issues or limitations due poor soft tissue coverage. Despite its overall limited success, the chances of improving results with a direct exchange procedure increases with the use of local antibiotic cement. One study indicated this success rate to be around $82 \%$ for cases using antibiotic cement compared to a rate of only $58 \%$ in cases that forgo its application ${ }^{14}$.

\section{Two-stage Exchange}

Two-stage exchange arthroplasty involves removal of the prosthesis and insertion of antibiotic impregnated cement spacer into the joint during the first stage of the procedure. Patient then is brought back for reimplantation once tissues are healed and infection is deemed to be eradicated ${ }^{15}$. This surgical option is the work horse of treating chronic PJI. Although historically this surgical intervention was believed to have success rate above $90 \%$ or so, in recent years the success of this procedure is believed to be in decline $e^{6}$. There is multitude of reasons that may explain the decline in success of this surgical procedure, the most important of which is the increase in the incidence of PJI caused by resistant organisms ${ }^{16}$. The incidence of such resistant organisms, like MRSA and MRSE, has doubled and quadrupled respectively, over the past decade ${ }^{9}$. Although PJI cases caused by MRSA are 9.2 times more likely to fail ${ }^{17}$, the two-stage exchange revision has been the best option thus far, as the failure rate of these cases is even greater with other treatments such as an irrigation and debridement where $70 \%$ result in failure ${ }^{11}$.

It is imperative for the success of this procedure, that careful monitoring of the infection take place at each stage of treatment. Aggressive debridement is a necessary during the first step, along with the removal of all foreign material, including any PMMA cement. A spacer laden with antibiotics is then put in place of the removed prosthesis. High doses of thermostable antibiotics should be used in the spacer. At our institution we utilize 4 grams of vancomycin and 3.6 grams of tobramycin in each 40 gram pack of PMMA. The patient is also given antibiotics for a minimum duration of 6 weeks postoperatively. 
The next stage, reimplantation, is advised when the serological markers, namely erythrocyte sedimentation rate (ESR) and C-reactive protein (CRP) returns to normal or had trended down considerably. In about $20 \%$ of PJI cases, the serological markers, in particular ESR, may still be abnormal at the time of reimplantation. Though desirable, the abnormal values do not prohibit the success of reimplantation significantly ${ }^{2}$. Prior to reimplantation, the joint may need to be aspirated in patients with abnormal serological markers or those suspected of harboring infection. At our institution any patients with previously failed two-stage exchange arthroplasty is aspirated prior to reimplantation arthroplasty, regardless of the results of serological markers. It is imperative that aspiration is performed when patient has been off the antibiotics for at least two weeks. When performed, the joint aspirate should be sent for neutrophil count and neutrophil percentage in addition to culture ${ }^{18}$.

During reimplantation it is critical that perioperative antibiotics is administered and if cemented components are used, antibiotic is also added to the cement. During reimplantation the traffic in the operating room needs to be controlled in a very strict fashion. Prior to reimplantation through debridement and irrigation of the joint needs to be performed and multiple tissue and fluid samples sent for culture. In patients who have undergone reimplantation and an organism is isolated from the intraoperative culture (even in broth), postoperative intravenous antibiotics needs to be administered.

In situations where the infection is uncontrollable, reimplantation is contraindicated. Furthermore, if the patient is medically unfit ( $8 \%$ to $10 \%$ of $\mathrm{PJI}$ patients), has neurological or vascular impairments, or a soft tissue deficiency persists, reimplantation is not recommended either. In general, results from our studies indicate that the true success rate of the two-stage exchange, is around $65-70 \%$, when success is defined as no infection, no mechanical failure or need for reoperation ${ }^{19}$.

\section{Salvage Procedures}

However rare, intricate and difficult cases do exist where reimplantation is not an option. These patients may have uncontrollable infections where salvage of the joint looks close to impossible. Additionally, they may be immunocompromised or they may have insufficient bone stock and muscle function necessary for successful joint reconstruction. The salvage operation in these cases is usually resection arthroplasty or in extremely rare circumstances disarticulation of the joint. Arthrodesis of the hip joint is almost never performed under these circumstances.

Patients with resection arthroplasty of the hip may be able to ambulate limited distances. Patients usually suffer an extensive limb length discrepancy and may have limited to no abductor function. Hip disarticulation may be performed for patients with uncontrollable infection and systemic manifestation of sepsis. It may also be employed in patients with extensive soft tissue deficiency in whom hygiene may be an issue. Patients with systemic sepsis and extensive osteomyelitis may also

require this salvage operation. Patients undergoing hind quarter amputation or hip disarticulation may not be able to ambulate.

\section{Conclusion}

For several decades total hip arthroplasty has increased the quality of life of patients receiving this procedure by reducing pain and increasing function of the arthritic hip joint. However, when infection 
follows a THA, it compromises the success of this procedure entirely. In order to successfully eradicate the infection and reconstruct the joint, it is imperative that the best treatment method is employed. An assortment of variables, specific to each case, shapes the viability of each treatment option in PJI cases. Thus, the success of any treatment option first rests in the hands of the clinician who integrates the surgical factors with those which evolve at the host-organism interface, in order to control and eradicate the infection.

\section{References:}

1. Bozic KJ, Kurtz SM, Lau E, Ong K, Chiu V, Vail TP, Rubash HE, Berry DJ. The epidemiology of revision total knee arthroplasty in the United States. Clin Orthop Relat Res. 2009

2. Donlan RM. Biofilms: microbial life on surfaces. Emerging Infect Dis. 2002; 8(9):881-890. : what are the diagnostic challenges? J Bone Joint Surg Am. 2006; 88(Suppl 4):138-147.

3. Davies DG, Parsek MR, Pearson JP, Iglewski BH, Costerton JW, Greenberg EP. The involvement of cell-to-cell signals in the development of a bacterial biofilm. Science. 1998; 280(5361):295-298.

4. Trampuz A, Osmon DR, Hanssen AD, Steckelberg JM, Patel R. Molecular and antibiofilm approaches to prosthetic joint infection. Clin Orthop Relat Res. 2003; (414):69-88.

5. Crockarell JR, Hanssen AD, Osmon DR, Morrey BF.Treatment of infection with debridement and retention of the components following hip arthroplasty. J Bone Joint Surg Am. 1998 Sep;80(9):1306-13.

6. Bradbury T, Fehring TK, Taunton M, et al. The fate of acute methicillin-resistant Staphylococcus aureus periprosthetic knee infections treated by open debridement and retention of components. J Arthroplasty. 2009; 24(6 Suppl):101-104.

7. Hartman MB, Fehring TK, Jordan L, Norton HJ. Periprosthetic knee sepsis. The role of irrigation and debridement. Clin Orthop Relat Res. 1991; (273):113-118.

8. Silva M, Tharani R, Schmalzried TP. Results of direct exchange or debridement of the infected total knee arthroplasty. Clin Orthop Relat Res. 2002; (404):125-131.

9. Parvizi J, Barrack RA Instr Course Lect 2008, Indicated that the incidence of MRSA and other resistant organisms is on the rise.

10. Sherrell JC, Fehring TK, Odum S, Hansen E, Zmistowski B, Dennos A, Kalore N; Periprosthetic Infection Consortium. The Chitranjan Ranawat Award: fate of two-stage reimplantation after failed irrigation and debridement for periprosthetic knee infection. Clin Orthop Relat Res. 2011 Jan;469(1):18-25.

11. Fehring T, Parvizi J, Barrack R, Hanssen AD. Fate of methicillin resistant infections. Clinical Orthopaedics and Related Research 2009

12. Mihalko WM, Manaswi A, Cui Q, Parvizi J, Schmalzried TP, Saleh KJ. Diagnosis and treatment of the infected primary total knee arthroplasty. Instr Course Lect. 2008;57:32739. Review. PubMed PMID: 18399596.

13. Raut VV, Siney PD, Wroblewski BM. One-stage revision of total hip arthroplasty for deep infection. Long-term followup. Clin Orthop. 1995; 321:202-207.

14. Garvin KL, Hanssen AD. Infection after total hip arthroplasty. Past, present, and future. J Bone Joint Surg Am. 1995; 77:1576-1588.

15. Ghanem E, Restrepo C, Joshi A, Hozack W, Sharkey P, Parvizi J. Periprosthetic infection does not preclude good outcome for revision arthroplasty. Clin Orthop Relat Res. 2007 Aug;461:54-9. PubMed PMID: 17572638. 
16. Parvizi J, Ghanem E, Menashe S, Barrack RL, Bauer TW. Periprosthetic infection: what are the diagnostic challenges? J Bone Joint Surg Am. 2006; 88(Suppl 4):138-147.

17. C. Salgado et al. Higher Risk of Failure of Methicillin-resistant Staphylococcus aureus Prosthetic Joint Infections. Clinical Orthopaedics and Related Research 2007; 461: 48-53

18. Parvizi J, Della Valle CJ. AAOS Clinical Practice Guideline: diagnosis and treatment of periprosthetic joint infections of the hip and knee. J Am Acad Orthop Surg. 2010 Dec;18(12):771-2.

19. Ghanem E, Restrepo C, Joshi A, Hozack W, Sharkey P, Parvizi J. Periprosthetic infection does not preclude good outcome for revision arthroplasty. Clin Orthop Relat Res. 2007 Aug;461:54-9. PubMed PMID: 17572638. 


\section{Disclosures}

Research Support:

NIH
OREF
DOD
Aircast
AOA
MTF
Stryker Orthopaedics
Pfizer
The Knee Society
Kimberly Clark
Ortho McNeill
Adolor
Cubist
3M
Zimmer
Biomemetics
Wyeth
Canadian Health
Board Member:
CD Diagnostics
Philadelphia Orthopedics
Eastern Orthopedics
United Healthcare

$\underline{\text { Consultant for: }}$

Stryker Orthopaedics

Zimmer

Biomet

Smith \& Nephew

Convatech

Intellectual Property:

SmarTech

CyruMed 